\title{
Architecture and Protocols for Satellite SDH Networks
}

\author{
Yanlang Hu, Quan Zhou, Jun Li, Yi Zhang, Jingling Li, and Tao Cui
}

\begin{abstract}
A novel architecture for a large amount of satellite data transport is presented which based on Synchronous Digital Hierarchy (SDH) technologies. This architecture consisting of terrestrial networks, satellites with an inter-satellite link (ISL), fixed earth stations, transportable earth stations, portable and handheld terminals, and user terminals connecting to satellite links directly or through terrestrial networks over SDH, over IP, over ATM or other network protocol. Datagrams such as ATM (asynchronous transfer mode) cells and IP (internet protocol) packets can be transported over SDH links between satellites in space. All kinds of ground terminals can access satellites directly with IP or ATM protocol or through SDH.
\end{abstract}

Index Terms-Digital Hierarchy (SDH), inter-satellite link (ISL), asynchronous transfer mode (ATM), Internet protocol (IP).

\section{INTRODUCTION}

Synchronous Digital Hierarchy (SDH) and Synchronous Optical Network (SONET) are time-division multiplexing technologies widely used in transport network. It is a standard for data transmission over telecommunication networks which are often used for framing and synchronization at the physical layer. SDH networks can carry large payloads, but, at the same time, accommodate lower speed signals packed into elementary transport units. Services such as videoconferencing, remote database access and multimedia file transfer require a flexible network with the availability (on demand) of virtually unlimited bandwidth. Satellites with SDH links seem highly suitable for globally delivery of these services. ITU-T has standardized the Synchronous Digital Hierarchy (SDH) as multiplexing technique between cross-connect or switches in the network. The compatibility of satellite with SDH is also analyzed by ITU-T and ITU-R in order to define the suitable data rates specified for SDH transmission over satellite systems. Several papers [1], [2] have addressed these special issues that SDH used in satellite scenarios. However, these papers mainly focus on the error performance monitoring defined by ITU-T and the path integrity from pointer corruption when busty errors occur.

In this paper a brief description of the SDH satellite network architectures and related protocol will be presented. The scheme proposed in his paper, based on SDH, combines two of popular protocols for satellites communication networks. It provides flexible access for various user

Manuscript received March 3, 2015; revised October 23, 2016. This work is supported by the National Natural Foundation of China (No.61372175) and the National Key Laboratory Foundation of China (No.9140C530403130C53192).

The authors are with National Key Laboratory of Science and Technology on Space Microwave, Xian 710100, China (e-mail: Huly39@cast504.com, zhouq@ cast504.com, Lij206@ sina.com, cuit81@ cast504.com). terminals such as fixed earth station, portable earth stations or handheld terminals with SDH interface, ATM interface or IP interface. Inter-satellite links are standard SDH links, on which STM-n frames are transported. Datagrams (ATM cells or IP packets) are encapsulated in STM-n on board.

\section{SATEllite Network ARChiteCtURE BASED ON SDH}

Fig. 1 illustrates a satellite SDH network represented by a ground segment, a space segment, and a network control center. The ground segment consists of ATM networks, IP networks that may be further connected to other legacy networks. The network control center (NCC) performs various management and resource allocation functions for the satellite media. Inter-satellite links (ISL), based on SDH, in the space segment provide seamless global connectivity to the satellite constellation.

The network allows the transmission of ATM cells, IP packets or other datagrams encapsulated in STM-16 over satellite, multiplexes and de-multiplexes STM-16 streams from uplinks and downlinks, and maintains the QoS objectives of the various connection types. The satellite-ATM interface and satellite SDH interface connect the ATM network and IP network to the satellite system respectively. The interface device transports ATM cells and IP packets over the frame based satellite SDH network, and demultiplexes ATM cells and IP packets from the satellite frames. This interface is thus responsible for resource allocation, error control, and traffic control.

ATM cells that transmitted by user terminals connected through ATM switches and IP packets transmitted by portable earth station and handheld terminal are encapsulated on board, while IP packets transmitted by user terminals connected through IP router are encapsulated by satellite SDH interface.

The basic SDH multiplexing techniques are described in ITU-TR Recs G.708 (1) and G.709 (2).The SDH multiplex hierarchy is based on a level 1 Frame structure (STM-1). Higher levels are also defined as STM-4 and STM- 16 formed by byte interleaving.

The inter-satellite links are based on high speed STM-16 frame, while these links between earth station and satellite are based on lower speed STM-4 frame or the basic STM-1 frame.

\section{IP OVER SATELLITE SDH NETWORKS}

IP is a network layer protocol, whose function is to permit data traffic to flow seamlessly between different types of transport mechanisms. IP resides in the terminal devices and in routers which function as switches in the network, routing packets towards their destination based on an address field 
contained in the datagram. IP-based applications, such as WWW and multimedia, have been an essential part of our life, and there is an ever-increasing demand for accessing high-speed Internet services anywhere and anytime [3]. In addition, with its broadcasting nature and global coverage, satellite systems also play an important role in the Internet. The proposed architecture in this paper provides access by user terminals in which IP resides.

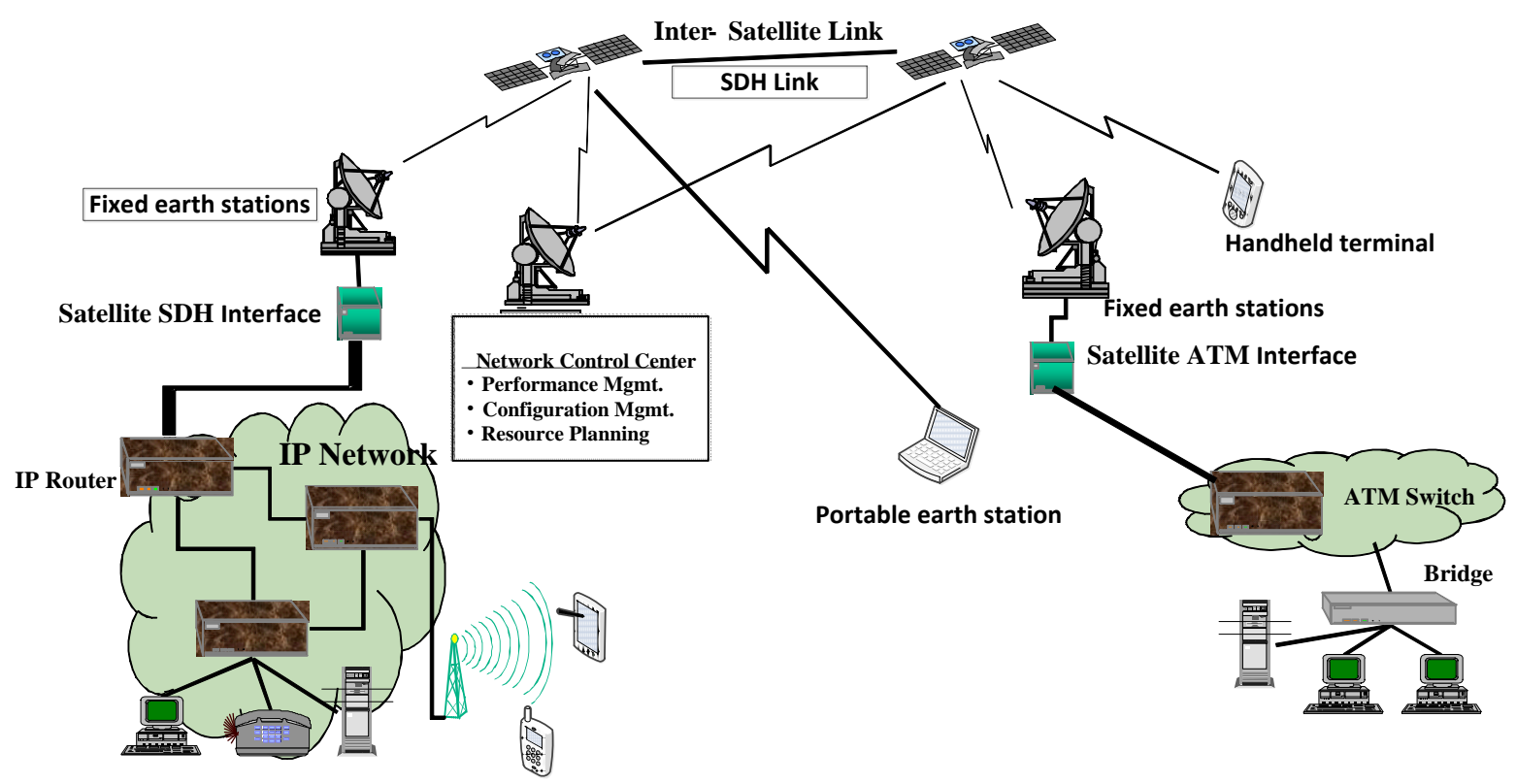

Fig. 1. Satellite-SDH network model.

End System

End System

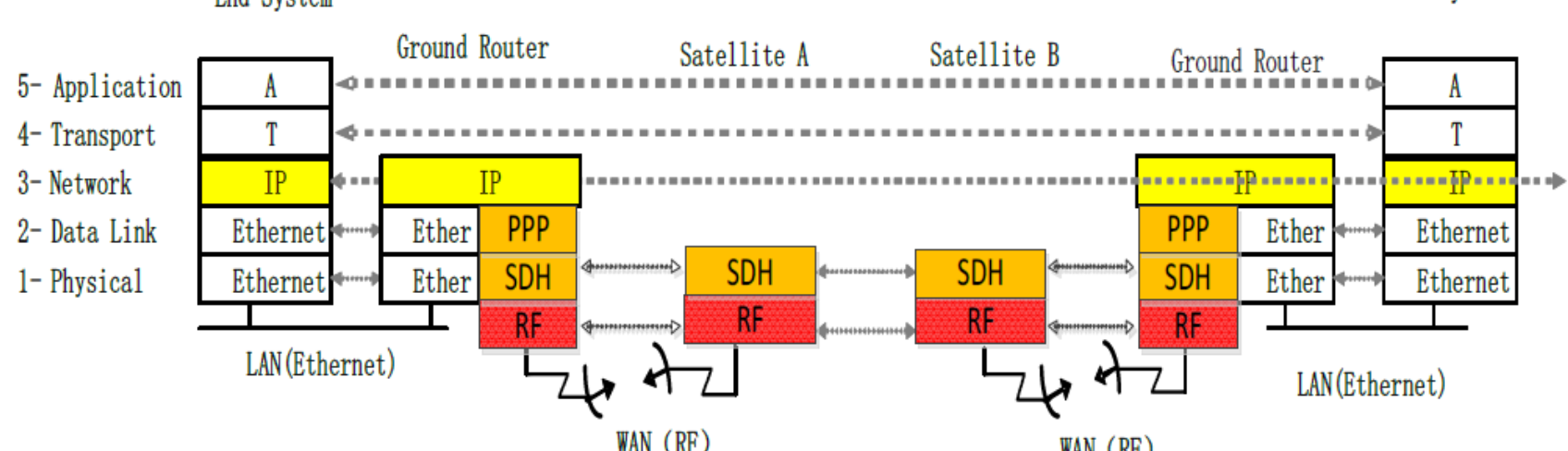

Fig. 2. IP over satellite SDH networks protocol stack diagram

\section{IP OVER SATELLITE SDH NETWORKS}

IP is a network layer protocol, whose function is to permit data traffic to flow seamlessly between different types of transport mechanisms. IP resides in the terminal devices and in routers which function as switches in the network, routing packets towards their destination based on an address field contained in the datagram. IP-based applications, such as WWW and multimedia, have been an essential part of our life and there is an ever-increasing demand for accessing high-speed Internet services anywhere and anytime [3]. In addition, with its broadcasting nature and global coverage, satellite systems also play an important role in the Internet. The proposed architecture in this paper provides access by user terminals in which IP resides.

Fig. 2 shows the protocol stack diagram used in end systems, ground routers which are parts of ground segment devices and SDH devices on board. The key points in this protocol stack is that the upper layer network services, layer 3 and above, operate on an end-to-end basis and are independent of the various physical media and data link protocols at layers 1 and 2. The end systems don't even know SDH at data link layer is in the path between the end users. Network systems, such as routers or bridges, between the end systems operate in only the lower 2 or 3 layers of the protocol stack and are completely unaware of what upper layer protocols are passing over them. This layered and modular approach is what allows the space networks to make changes and upgrades on some stations without any impact on other stations.

IP packet encapsulation is an aspect for IP over SDH network. It is a technique used to encapsulate an IP packet into the STM-1 frame, so that it is suitable for transmission over the satellite SDH network. In fact, PPP is the data link protocol used in IP over SDH. It is a connection-oriented protocol that enables layer two links over a variety of different physical layer connections. It was designed to carry IP traffic but is general enough to allow any type of network layer datagram to be sent over a PPP connection. 


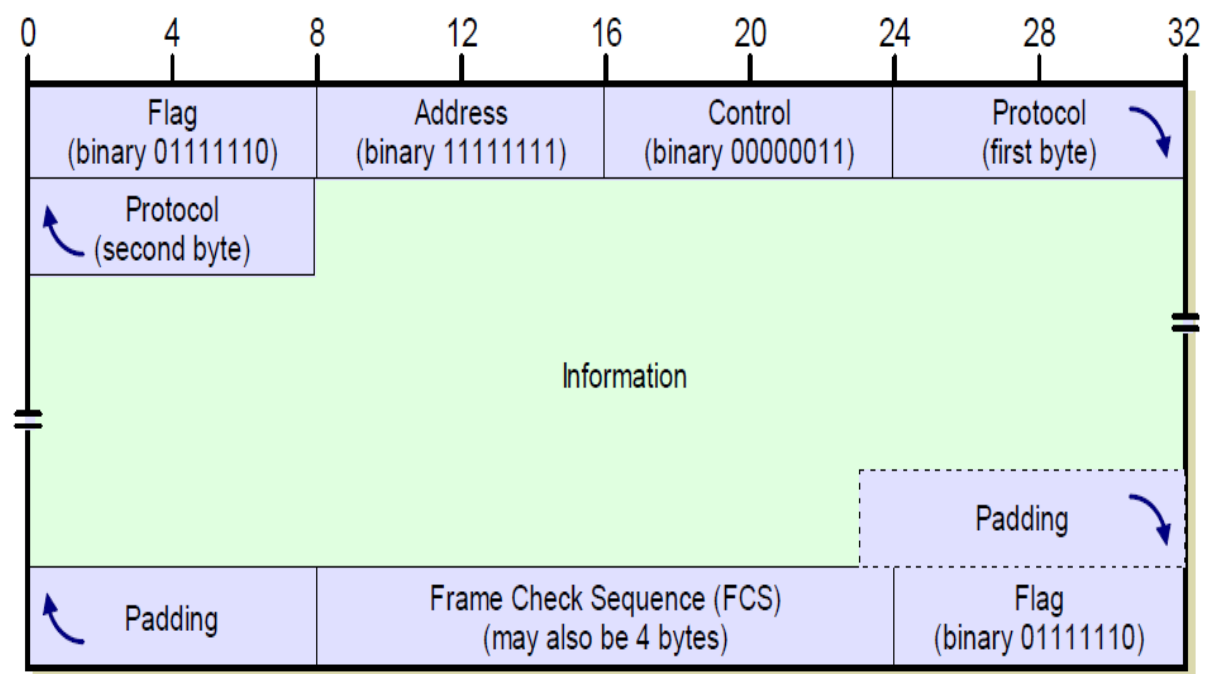

Fig. 3. PPP frame format.

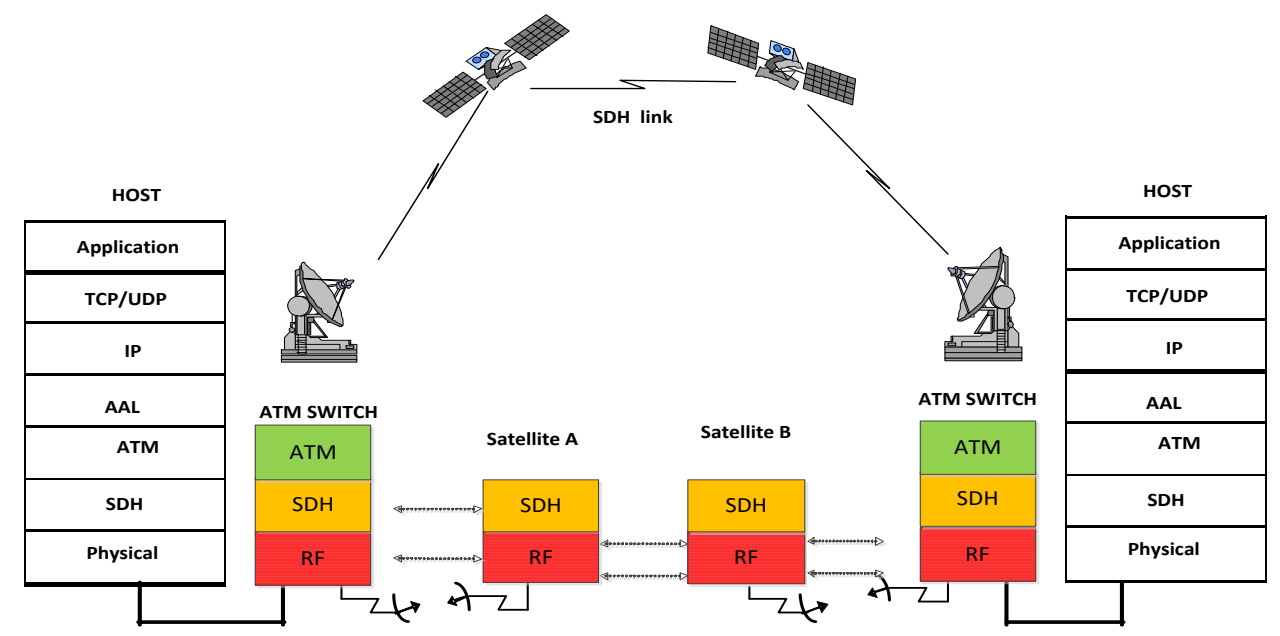

Fig. 4. ATM over SDH protocol stack diagram.

Fig. 3 shows the application of the PPP general frame format which carries IP data. The value 0x0021 in the Protocol field indicates this as an IPv4 datagram. Flag field Indicates the start of a PPP frame. Always has the value "01111110"binary.Address field has no real meaning. It is thus always set to "11111111" (0xFF or 255 decimal), which is equivalent to a broadcast (it means "all stations").Control field is used in HDLC for various control purposes, but in PPP it is set to "00000011" (3 decimal).

In brief, the transmission of IP over SDH is as follows: first, IP datagrams are encapsulated in PPP frame, then, PPP frame is mapped into SDH virtual containers (VC) for transmission.

\section{ATM OVER SATELLITE SDH NeTWORKS}

ATM technology is an ITU-T standard for cell relay wherein information for multiple service types, such as voice, video, or data, is conveyed in small, fixed-size cells. ATM networks are connection oriented. It is a cell-switching and multiplexing technology that combines the benefits of circuit switching (guaranteed capacity and constant transmission delay) with those of packet switching (flexibility and efficiency for intermittent traffic). It provides scalable bandwidth from a few megabits per second (Mbps) to many gigabits per second (Gbps). It is expected to provide quality of service based networks that support voice, video and data applications. ATM based satellite networks effectively provide real time as well as non-real time communications services to remote areas [4]. The proposed network architecture based SDH also provides access by ATM terminals.

As Fig. 4 shows ATM over SDH protocol stack is very similar to IP over SDH. The key difference is interface devices that match different datagram into SDH basic frame. Only SDH protocol is processed on board satellite as backbone transport link. This approach reduces the complexity of on board processing, and gains advantages of ATM in satellite communications.

The ITU-T defined the approach to transmit ATM cells over SDH. The essential feature of SDH is to keep track of boundaries of streams that do not really depend on the particular medium. Although it was originally designed for transmission over fiber, it can in fact operate over satellite media. The SDH mode type 1 (STM-1) frame [5], as shown in Fig. 5 is 155 Mbit/s. The bytes are transmitted across the medium a row at a time, wrapping to the next row. It takes nominally 125 microseconds to transmit all nine rows forming the SDH STM-1 frame. The STM-1 in the international carrier networks will be the smallest package available in terms of the SDH. 


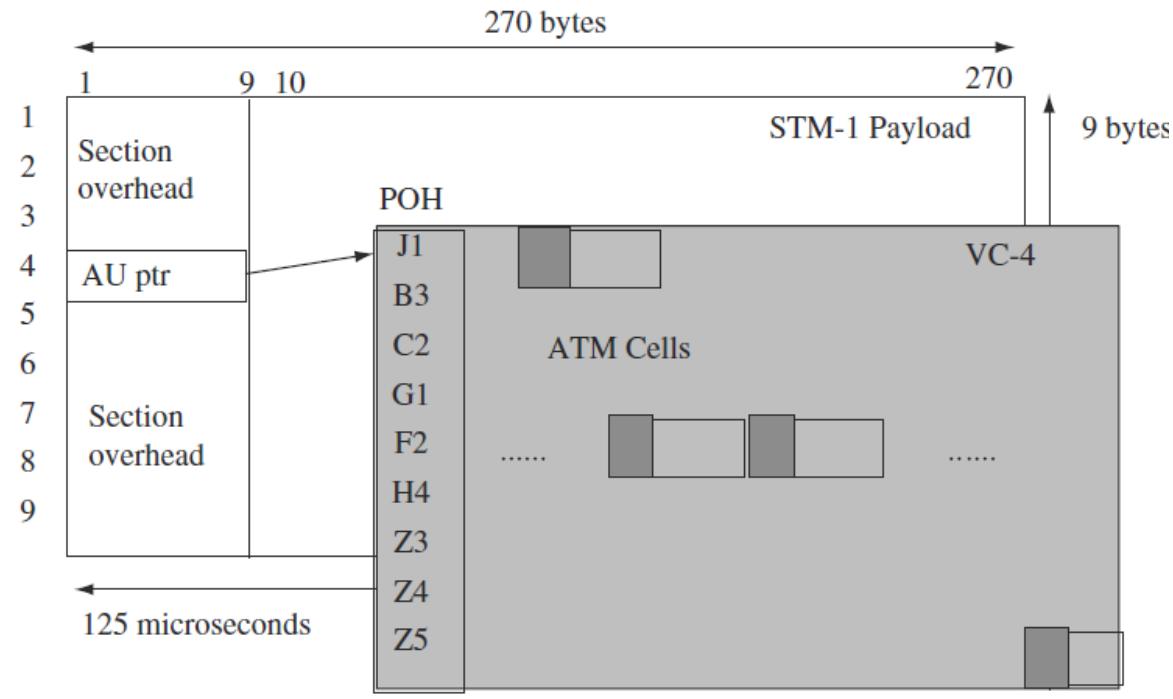

Fig. 5. Mapping ATM cells in STM-1.

Using the header error check (HEC) of the ATM cell delineates the cells within the SDH payload (VC-4 container). The receiver takes five bytes to check if they form a header or not when it is trying to find the cell boundaries. HEC calculation, matching against the fifth byte, is done on the first four bytes. If it matches, the receiver then counts 48 bytes and repeats the calculation again. And if it finds that calculation correct several times in a row, it can be safely assumed that the cell boundaries are found. If it fails to match, it just slides the window by one bit and tries the calculation again. This kind of process must be used because the user data would contain these patterns separated by 48 bytes. The payload in an STM-1 frame is $135,563 \mathrm{Mbit} / \mathrm{s}$, assuming that the entire cell payload may carry user information.

\section{CONCLUSION}

A SDH based satellites network architecture is presented in this article. In this architecture, only SDH protocol is processed on board, all other layer 3 and layer 2 protocols is processed on ground. This approach reduces the complexity of satellite on board processing greatly, and is expected to provide quality of service (QoS) based on the architecture that supports voice, video and data applications. Along with the architecture, two popular communication protocols,
ATM and IP, over the proposed SDH satellites network are presented. The two protocol stacks can encapsulate ATM cells and IP packets in STM-1 frame properly and provide access for ATM terminals and IP terminals on ground.

\section{REFERENCES}

[1] A. Strange, S. L. Arambepola, A. J. FIavin, and A. Stevenson, "SDH for the fixed satellite service," The Institution of Electrical Engineers, 1994.

[2] R. C. Lou, "IPv6 networks over DVB-RCS satellite systems," International Journal of Satellite Communications and Networking, 2007.

[3] W. D. Ivancic, "NASA's broadband satellite networking research," IEEE Communications Magazine, 1999.

[4] Z. L. Sun, "Satellite networking: principles and protocols," pp 187-212, 2005.

[5] RFC 1122, "Requirements for internet hosts - Communication layers," p. 18, 2010.

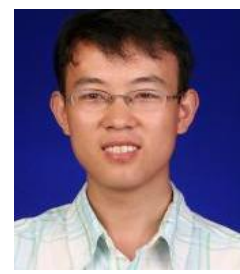

Yanlang hu was born in Yan'an of China in 1981. He received the diploma in communication and system engineering from China Academy of Space Technology in 2008. His research interests include onboard switching techniques, digital image processing, machine vision, and machine learning. 